\title{
RADIATIVE FALKNER-SKAN FLOW OF WALTER-B FLUID WITH PRESCRIBED SURFACE HEAT FLUX
}

\author{
TASAWAR HAYAT \\ Department of Mathematics, Quaid-I-Azam University, Islamabad, Pakistan and \\ Nonlinear Analysis and Applied Mathematics (NAAM) Research Group, Department of Mathematics, \\ King Abdulaziz University, Jeddah, Saudi Arabia \\ Sumaira Qayyum, Maria Imtiaz \\ Department of Mathematics, Quaid-I-Azam University, Islamabad, Pakistan; e-mail: mi_qau@yahoo.com \\ Ahmed Alsaedi \\ Nonlinear Analysis and Applied Mathematics (NAAM) Research Group, Department of Mathematics, \\ King Abdulaziz University, Jeddah, Saudi Arabia
}

\begin{abstract}
This article addresses the Falkner-Skan flow of an incompressible Walter-B fluid. Fluid flow is caused by a stretching wedge with thermal radiation and prescribed surface heat flux. Appropriate transformations are used to obtain the system of nonlinear ordinary differential equations. Convergent series solutions are obtained by the homotopy analysis method. Influence of pertinent parameters on the velocity, temperature and Nusselt number are investigated. It is observed that by increasing the viscoelastic parameter, the fluid velocity decreases. There is an enhancement of the heat transfer rate for the viscoelastic parameter and power law index. It is also found that the Prandtl number and radiation parameter decrease the heat transfer rate.
\end{abstract}

Keywords: Walter-B fluid, Falkner-Skan flow, prescribed surface heat flux, thermal radiation

\section{Introduction}

Non-Newtonian materials in view of its complex constitutive expression yield much more complicated and higher order differential systems when compared with viscous materials. Such complexities in differential systems are due to additional rheological parameters appearing in the constitutive relationships. Even a simpler constitutive equation like for Walter-B gives rise to nonlinear boundary initial value problems which are far from trivial. These boundary value problems have great interest of researchers from different quarters. For example Chang et al. (2011) numerically analyzed the free convective heat transfer in viscoelastic flow of Walter-B fluid. Nadeem et al. (2015) examined oblique flow of Walter-B fluid in presence of magnetohydrodynamics and nanoparticles. Nandeppanavar et al. (2010) explored stretched flow of Walter-B liquid in presence of non-uniform heat source/sink. Hakeem et al. (2014) extended such analysis in presence of thermal radiation. Stagnation point flow and Blasius flow for Walter-B liquid were also addressed by Madani et al. (2012). Hayat et al. (2014a, 2015c) examined heat transfer in flow of Walter-B fluid over a surface with Newtonian heating and convective condition. Talla (2013) studied the flow of Walter-B fluid bounded by an exponentially stretching sheet. Peristalsis of Walter-B fluid in a vertical channel was studied by Ramesh and Devakar (2015).

Falkner-Skan flow is quite popular in fluid mechanics. It is a flow past a wedge placed symmetrically with respect to the flow direction. These types of flows occur frequently to increase 
oil recovery and in packed bed reactor geothermal industries. Interest of recent researchers in boundary layer flow over a continuos moving surface with prescribed surface heat flux has increased so much. These type of flows have many applications in industrial and metallurgical processes such as glass fiber, wire drawing, paper production and metallic plate cooling in cooling bath, etc. Falkner and Skan (1931) presented some approximate solutions for the boundary layer equation. Yacob et al. (2011) studied the Falkner-Skan problem for a static and moving wedge with prescribed surface heat flux in a nanofluid. Falkner-Skan flow of the Maxwell fluid with mixed convection was analyzed by Hayat et al. (2012). Khan and Pop (2013) examined the nanofluid flow past a moving wedge. Abbasbandy et al. (2014b) discussed numerical and analytical solutions for MHD Falkner-Skan flow of the Maxwell fluid. Hendi and Hussain (2012) found the solution for MHD Falkner-Skan flow over a permeable sheet. Fang et al. (2012) studied the momentum and heat transfer in Falkner-Skan flow with algebraic decay. Su and Zheng (2011) presented the approximate solution of MHD Falkner-Skan flow over a permeable wall. Abbasbandy et al. (2014a) worked for Falkner-Skan flow of an Oldroyd-B fluid in presence of the applied magnetic field.

The radiation effect in boundary layer flow has much importance due to its applications in physics, engineering and industrial fields such as glass production, furnace design, polymer processing, gas cooled nuclear reactors and also in space technology like aerodynamics of rockets, missiles, propulsion system, power plants for inter planetary fights and space crafts operating at high temperatures. Heat transfer through radiation takes place in form of electromagnetic waves. Radiation emitted by a body is a consequence of thermal agitation of its composing molecules. Hayat et al. (2013c) worked on mixed convection radiative stagnation point flow in presence of convective boundary conditions. Hayat et al. (2013b) also discussed the effect of thermal radiation in MHD flow of thixotropic fluid. Pal (2013) analyzed the effects of thermal radiation, Hall current and MHD in flow over an unsteady stretching surface. Bhattacharyya et al. (2012) analyzed the flow of micropolar fluid over a porous shrinking sheet with thermal radiation. Hayat et al. (2013a)studied the three-dimensional MHD flow of Eyring-Powell fluid with radiative effects. Rashidi et al. (2014) discussed the influence of thermal radiation in MHD mixed convective flow of a viscoelastic fluid due to a porous wedge. Bhattacharyya (2013) presented the MHD Casson fluid subject to thermal radiation. Sheikholeslami et al. (2015) adopted a two phase model for MHD flow of a nanofluid with thermal radiation.

The aim of present study is to venture further in the region of Falkner-Skan flow of a non-Newtonian fluid. Thus flow formulation here is based upon constitutive relationship of WaltersB fluid. Analysis of heat transfer is carried out in presence of heat flux and thermal radiation. Transformation procedure has been used for the reduction of partial differential systems to ordinary differential systems. The homotopy analysis technique has been implemented for the development of convergent series solutions. Influences of pertinent parameters on the velocity, temperature and Nusselt number are pointed out.

\section{Problem formulation}

We consider the steady two-dimensional Falkner-Skan flow of an incompressible Walter-B fluid. Heat transfer analysis is carried out in the presence of prescribed surface heat flux and thermal radiation. The fluid flow is induced via stretching a wedge moving with the velocity $U_{w}=c x^{n}$ and the fluid flow being confined to $y \geqslant 0$. Let $T_{\infty}$ be ambient temperature. The relevant boundary layer equations are (Hakeem et al., 2014) 


$$
\begin{aligned}
& \frac{\partial u}{\partial x}+\frac{\partial v}{\partial y}=0 \\
& u \frac{\partial u}{\partial x}+v \frac{\partial u}{\partial y}=U_{e} \frac{d U_{e}}{d x}+\nu \frac{\partial^{2} u}{\partial y^{2}}-k_{0}\left(u \frac{\partial^{3} u}{\partial x \partial y^{2}}+v \frac{\partial^{3} u}{\partial y^{3}}+\frac{\partial u}{\partial x} \frac{\partial^{2} u}{\partial y^{2}}-\frac{\partial u}{\partial y} \frac{\partial^{2} u}{\partial y \partial x}\right) \\
& u \frac{\partial T}{\partial x}+v \frac{\partial T}{\partial y}=\frac{k}{\rho c_{p}} \frac{\partial^{2} T}{\partial y^{2}}-\frac{1}{\rho c_{p}} \frac{\partial q_{r}}{\partial y}
\end{aligned}
$$

The corresponding boundary conditions are (Yacob et al., 2011)

$$
\begin{array}{llll}
u=U_{w}=c x^{n} & v=0 & \frac{\partial T}{\partial y}=-\frac{q_{w}}{k} \quad \text { at } \quad y=0 \\
u \rightarrow U_{e}=a x^{n} & T \rightarrow T_{\infty} & \text { as } y \rightarrow \infty &
\end{array}
$$

where $(u, v)$ are the velocities along $(x, y)$ directions respectively, $T$ is temperature, $\nu$ is kinematic viscosity, $k_{0}$ is elastic parameter, $k$ is thermal conductivity, $\rho$ is density, $c_{p}$ is specific heat, $c$ and $a$ are the stretching rates and $q_{w}$ the wall heat flux. Radiative heat flux by using Rosseland approximation is given by

$$
q_{r}=-\frac{4 \sigma^{*}}{3 k^{*}} \frac{\partial T^{4}}{\partial y}
$$

where $\sigma^{*}$ is the Stefan-Boltzmann constant and $k^{*}$ the mean absorption coefficient. Further, we assume that the temperature difference within the flow is such that $T^{4}$ may be expanded in a Taylor series. Hence expanding $T^{4}$ about $T_{\infty}$ and neglecting higher order terms, we get

$$
T^{4} \cong 4 T_{\infty}^{3} T-3 T_{\infty}^{4}
$$

Using Eqs. (2.3) and (2.4) in (2.1) 3 , we obtain

$$
u \frac{\partial T}{\partial x}+v \frac{\partial T}{\partial y}=\frac{1}{\rho c_{p}}\left(k+\frac{16 \sigma^{*} T_{\infty}^{3}}{3 k^{*}}\right) \frac{\partial^{2} T}{\partial y^{2}}
$$

Suitable transformations for the present flow are (Yacob et al., 2011)

$$
\begin{array}{rlrl}
\eta & =\sqrt{\frac{n+1}{2}} \sqrt{\frac{U_{e}}{\nu x}} y & v & =-\sqrt{\frac{n+1}{2}} \sqrt{\frac{\nu U_{e}}{x}}\left(f(\eta)+\frac{n-1}{n+1} \eta f^{\prime}(\eta)\right) \\
u & =a x f^{\prime}(\eta) & \theta & =\frac{k\left(T-T_{\infty}\right)}{q_{w}} \sqrt{\frac{(n+1) U_{e}}{2 \nu x}}
\end{array}
$$

where $x$ is the distance from the leading edge and $n$ the Falkner-Skan power-law parameter. Using Eq. (2.3), the continuity equation is satisfied automatically and Eqs. $(2.1)_{2}-(2.2)$ take the form

$$
\begin{aligned}
& f^{\prime \prime \prime}+f f^{\prime \prime}+\frac{2 n}{n+1}\left(1-f^{\prime 2}\right)-k_{1}\left((3 n-1) f^{\prime} f^{\prime \prime \prime}-\frac{n+1}{2} f f^{\prime \prime \prime \prime}-\frac{3 n-1}{2} f^{\prime \prime 2}\right)=0 \\
& \frac{1}{\operatorname{Pr}}\left(1+\frac{4}{3} R\right) \theta^{\prime \prime}+f \theta^{\prime}+\frac{n-1}{n+1} f^{\prime} \theta=0
\end{aligned}
$$

and

$$
\begin{array}{lrl}
f(0)=0 & f^{\prime}(\infty) \rightarrow 1 & f^{\prime}(0)=\alpha \\
\theta^{\prime}(0)=-1 & \theta(\infty) \rightarrow 0 &
\end{array}
$$

where $k_{1}$ is the viscoelastic parameter, $\operatorname{Pr}$ is the Prandtl number, $\alpha$ is the ratio of stretching rates and $R$ is the radiation parameter. The dimensionless parameters are defined as follows

$$
k_{1}=\frac{k_{0} a x^{n-1}}{\rho \nu} \quad \operatorname{Pr}=\frac{\rho c_{p} \nu}{k} \quad \alpha=\frac{c}{a} \quad R=\frac{4 \sigma^{*} T_{\infty}^{3}}{k k^{*}}
$$


The local Nusselt number in the dimensional form is

$$
\mathrm{Nu}_{x}=\frac{x q_{w}}{k\left[\frac{q_{w}}{k}\left(\frac{2 \nu x}{n+1}\right) U_{e}(x)\right]}
$$

with

$$
q_{w}=-\left.k \frac{\partial T}{\partial y}\right|_{y=0}+\left(q_{r}\right)_{w}
$$

in which $q_{r}$ is prescribed as follows

$$
\left(q_{r}\right)_{w}=\left.\frac{-16 \sigma^{*} T_{\infty}^{3}}{3 k^{*}} \frac{\partial T}{\partial y}\right|_{y=0}
$$

The dimensionless form of the Nusselt number is

$$
\frac{\mathrm{Nu}_{x}}{\sqrt{\mathrm{Re}_{x}}}=-\sqrt{\frac{n+1}{2}}\left(1+\frac{4 R}{3}\right) \theta^{\prime}(0)
$$

\section{Homotopic solutions}

\subsection{Zeroth-order deformation equations}

Initial approximations and auxiliary linear operators are taken as follows

$$
\begin{aligned}
& f_{0}(\eta)=\eta-(1-\alpha)[1-\exp (-\eta)] \quad \theta_{0}(\eta)=\exp (-\eta) \\
& \mathrm{L}_{f}=f^{\prime \prime \prime}-f^{\prime} \quad \mathrm{L}_{\theta}=\theta^{\prime \prime}-\theta
\end{aligned}
$$

with

$$
\mathrm{L}_{f}\left[c_{1}+c_{2} \mathrm{e}^{\eta}+c_{3} \mathrm{e}^{-\eta}\right]=0 \quad \mathrm{~L}_{\theta}\left[c_{4} \mathrm{e}^{\eta}+c_{5} \mathrm{e}^{-\eta}\right]=0
$$

where $c_{i}(i=1-5)$ are constants.

Denoting $q \in[0,1]$ as the embedding parameter and $\hbar_{f}$ and $\hbar_{\theta}$ as the non-zero auxiliary parameters, then the zeroth order deformation problems are

$$
\begin{aligned}
& (1-q) \mathrm{L}_{f}\left[F(\eta, q)-f_{0}(\eta)\right]=q \hbar_{f} N_{f}[F(\eta, q)] \\
& (1-q) \mathrm{L}_{\theta}\left[\vartheta(\eta, q)-\theta_{0}(\eta)\right]=q \hbar_{\theta} N_{\theta}[\vartheta(\eta, q), F(\eta, q)] \\
& F(0, q)=0 \quad F^{\prime}(0, q)=\alpha \quad F^{\prime}(\infty, q)=1 \\
& \vartheta^{\prime}(0, q)=-1 \quad \vartheta(\infty, q)=0
\end{aligned}
$$

where the nonlinear differential operators $\mathrm{N}_{f}$ and $\mathrm{N}_{\theta}$ are

$$
\begin{aligned}
& \mathrm{N}_{f}[F(\eta, q)]=\frac{\partial^{3} F(\eta, q)}{\partial \eta^{3}}+\frac{2 n}{n+1}\left[1-\left(\frac{\partial F(\eta, q)}{\partial \eta}\right)^{2}\right]+F(\eta, q) \frac{\partial^{2} F(\eta, q)}{\partial \eta^{2}} \\
& \quad-k_{1}\left[(3 n-1) \frac{\partial F(\eta, q)}{\partial \eta} \frac{\partial^{3} F(\eta, q)}{\partial \eta^{3}}-\frac{n+1}{2} F(\eta, q) \frac{\partial^{4} F(\eta, q)}{\partial \eta^{4}}-\frac{3 n-1}{2}\left(\frac{\partial^{2} F(\eta, q)}{\partial \eta^{2}}\right)^{2}\right] \\
& \mathrm{N}_{\theta}[\vartheta(\eta, q), F(\eta, q)]=\frac{1}{\operatorname{Pr}}\left(1+\frac{4}{3} R\right) \frac{\partial^{2} \vartheta(\eta, q)}{\partial \eta^{2}}+\frac{\partial \vartheta(\eta, q)}{\partial \eta} F(\eta, q) \\
& \quad+\frac{n-1}{n+1} \frac{\partial F(\eta, q)}{\partial \eta} \vartheta(\eta, q)
\end{aligned}
$$




\section{2. $m$-th order deformation equations}

The $m$-th order deformation problems are

$$
\mathrm{L}_{f}\left[f_{m}(\eta)-\chi_{m} f_{m-1}(\eta)\right]=\hbar_{f} \mathrm{R}_{f, m}(\eta) \quad \mathrm{L}_{\theta}\left[\theta_{m}(\eta)-\chi_{m} \theta_{m-1}(\eta)\right]=\hbar_{\theta} \mathrm{R}_{\theta, m}(\eta)
$$

and

$$
f_{m}(0)=\frac{\partial f_{m}(0)}{\partial \eta}=\frac{\partial f_{m}(\infty)}{\partial \eta}=\theta^{\prime}(0)=\theta(\infty)=0
$$

where $\mathrm{R}_{f, m}(\eta)$ and $\mathrm{R}_{\theta, m}(\eta)$ have the following forms

$$
\begin{aligned}
& \mathrm{R}_{f, m}(\eta)=f_{m-1}^{\prime \prime \prime}+\frac{2 n}{n+1}\left(1-\sum_{k=0}^{m-1} f_{m-1-k}^{\prime} f_{k}^{\prime}\right)+\sum_{k=0}^{m-1} f_{m-1-k} f_{k}^{\prime \prime} \\
&-k_{1}\left[(3 n-1) f_{m-1-k}^{\prime} f_{k}^{\prime \prime \prime}-\frac{n+1}{2} \sum_{k=0}^{m-1} f_{m-1-k} f_{k}^{\prime \prime \prime \prime}-\frac{3 n-1}{2} \sum_{k=0}^{m-1} f_{m-1-k}^{\prime \prime} f_{k}^{\prime \prime}\right] \\
& \mathrm{R}_{\theta, m}(\eta)=\frac{1}{\operatorname{Pr}}\left(1+\frac{4}{3} R\right) \theta_{m-1}^{\prime \prime}+\sum_{k=0}^{m-1} f_{m-1-k} \theta_{k}^{\prime}+\frac{n-1}{n+1} \sum_{k=0}^{m-1} f_{m-1-k}^{\prime} \theta_{k}
\end{aligned}
$$

and

$$
\chi_{m}=\left\{\begin{array}{lll}
0 & \text { for } & m \leqslant 1 \\
1 & \text { for } & m>1
\end{array}\right.
$$

The general solutions $\left(f_{m}, \theta_{m}\right)$ comprising the special solutions $\left(f_{m}^{*}, \theta_{m}^{*}\right)$ are

$$
\begin{aligned}
& f_{m}(\eta)=f_{m}^{*}(\eta)+c_{1}+c_{2} \mathrm{e}^{\eta}+c_{3} \mathrm{e}^{-\eta} \\
& \theta_{m}(\eta)=\theta_{m}^{*}(\eta)+c_{4} \mathrm{e}^{\eta}+c_{5} \mathrm{e}^{-\eta}
\end{aligned}
$$

where the constants $c_{i}(i=1-5)$ through boundary conditions (3.6) are

$$
\begin{array}{ll}
c_{1}=-c_{3}-f_{m}^{*}(0) & c_{3}=\frac{\partial f_{m}^{*}(0)}{\partial \eta} \\
c_{5}=\frac{\partial \theta_{m}^{*}(0)}{\partial \eta} & c_{2}=c_{4}=0
\end{array}
$$

\section{Convergence analysis}

The homotopy analysis method has great advantage to adjust the convergence region by selecting the appropriate values of $\hbar_{f}$ and $\hbar_{\theta}$. For this, we plot the $\hbar$-curves for the convergence of velocity and temperature profiles (see Fig. 1). Admissible values of auxiliary parameters are $-0.9 \leqslant \hbar_{f} \leqslant 0$ and $-0.6 \leqslant \hbar_{\theta} \leqslant-0.2$. The solution converges in the whole region of $\eta$ $(0 \leqslant \eta \leqslant \infty)$ when $k_{1}=0.2, n=0.1, R=1.6, \operatorname{Pr}=1.5$ and $\alpha=0.9$.

Table 1 shows the convergence of functions $f^{\prime \prime}(0)$ and $\theta^{\prime \prime}(0)$ at a different order of approximations. Tabulated values show that the 25-th order of approximations is enough for the convergence of $f^{\prime \prime}(0)$, and the 22-th order of approximation is appropriate for the convergence of $\theta^{\prime \prime}(0)$. 


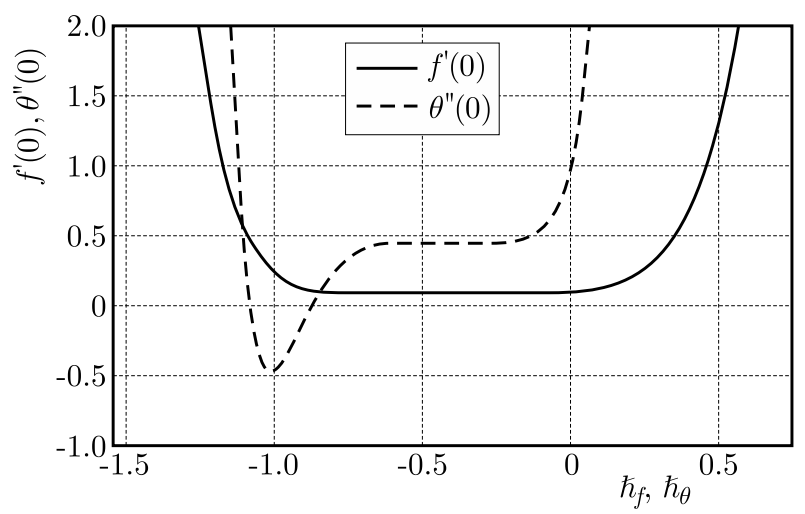

Fig. 1 . $\hbar$-curves for $f^{\prime \prime}(0)$ and $\theta^{\prime \prime}(0)$ when $k_{1}=0.2, n=0.1, R=1.6$, Pr $=1.5$ and $\alpha=0.9$.

Table 1. Convergence of HAM (homotopy analysis method) solutions when $k_{1}=0.2, n=0.1$, $R=1.6, \operatorname{Pr}=1.5, \alpha=0.9, \hbar_{f}=-0.2=\hbar_{\theta}$

\begin{tabular}{|c|c|c|}
\hline $\begin{array}{c}\text { Order of } \\
\text { approximation }\end{array}$ & $f^{\prime \prime}(0)$ & $\theta^{\prime \prime}(0)$ \\
\hline \hline 1 & 0.09734 & 0.8154 \\
\hline 5 & 0.09262 & 0.8203 \\
\hline 10 & 0.09262 & 0.4547 \\
\hline 15 & 0.09171 & 0.4454 \\
\hline 20 & 0.09168 & 0.4447 \\
\hline 22 & 0.09169 & 0.4446 \\
\hline 25 & 0.09170 & 0.4446 \\
\hline 30 & 0.09170 & 0.4446 \\
\hline 35 & 0.09170 & 0.4446 \\
\hline 40 & 0.09170 & 0.4446 \\
\hline 45 & 0.09170 & 0.4446 \\
\hline
\end{tabular}

\section{Discussion}

In this Section, we discussed the influences of different physical parameters on the fluid velocity, temperature and heat transfer rate.

\subsection{Dimensionless velocity profile}

Figures 2a-2c show the effect of viscoelastic parameter $k_{1}$, power law index $n$ and stretching rates ratio $\alpha$ on the velocity profile. Figure 2a depicts the influence of the viscoelastic parameter on $f^{\prime}(\eta)$. As $k_{1}$ increases, the fluid velocity decreases which corresponds to a thinner momentum boundary layer thickness. The viscoelasticity produces tensile stress which contracts the boundary layer and, consequently, the velocity reduces. Figure $2 \mathrm{~b}$ represents the impact of $\alpha$ on the velocity profile. Here, the velocity enhances by increasing $\alpha$. In fact higher values of $\alpha$ correspond to the stronger free stream velocity which enhances the fluid velocity. The effect of Falkner-Skan power law index $n$ is graphed in Fig. 2c. It is observed that velocity is an increasing function of $n$.

\subsection{Dimensionless temperature profile}

Figures 3 and 4 show the impact of the Prandtl number Pr, radiation parameter $R$, viscoelastic parameter $k_{1}$, power law index $n$ and ratio of stretching rates $\alpha$ on the temperature profile. 
(a)

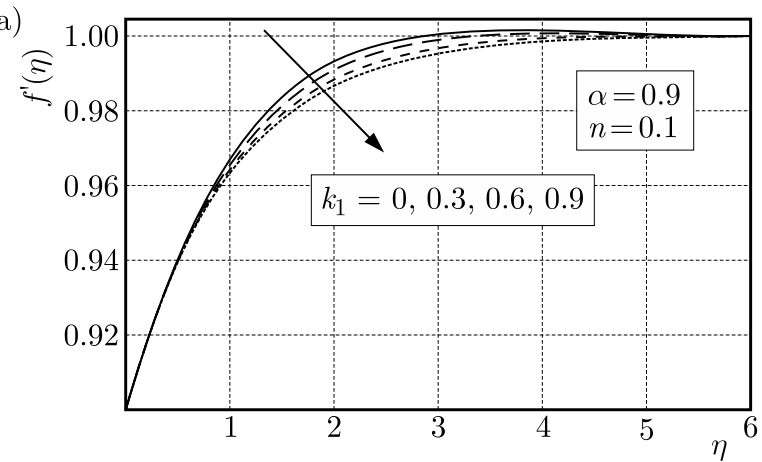

(b)

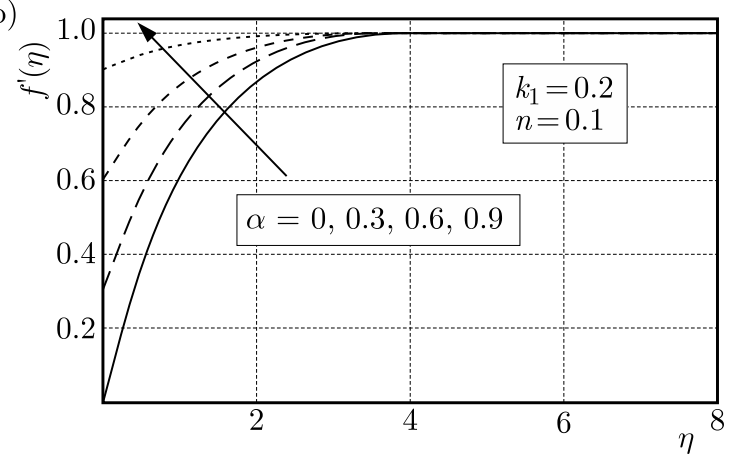

(c)

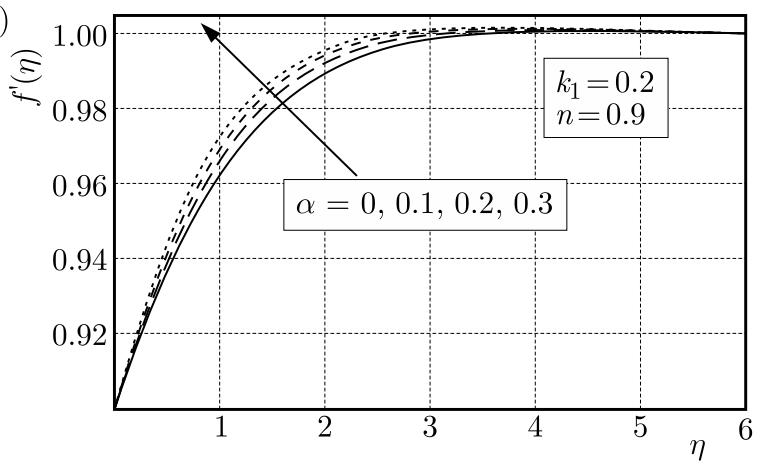

Fig. 2. Impact of: (a) $k_{1}$, (b) $\alpha$ and (c) $n$ on $f^{\prime}(\eta)$
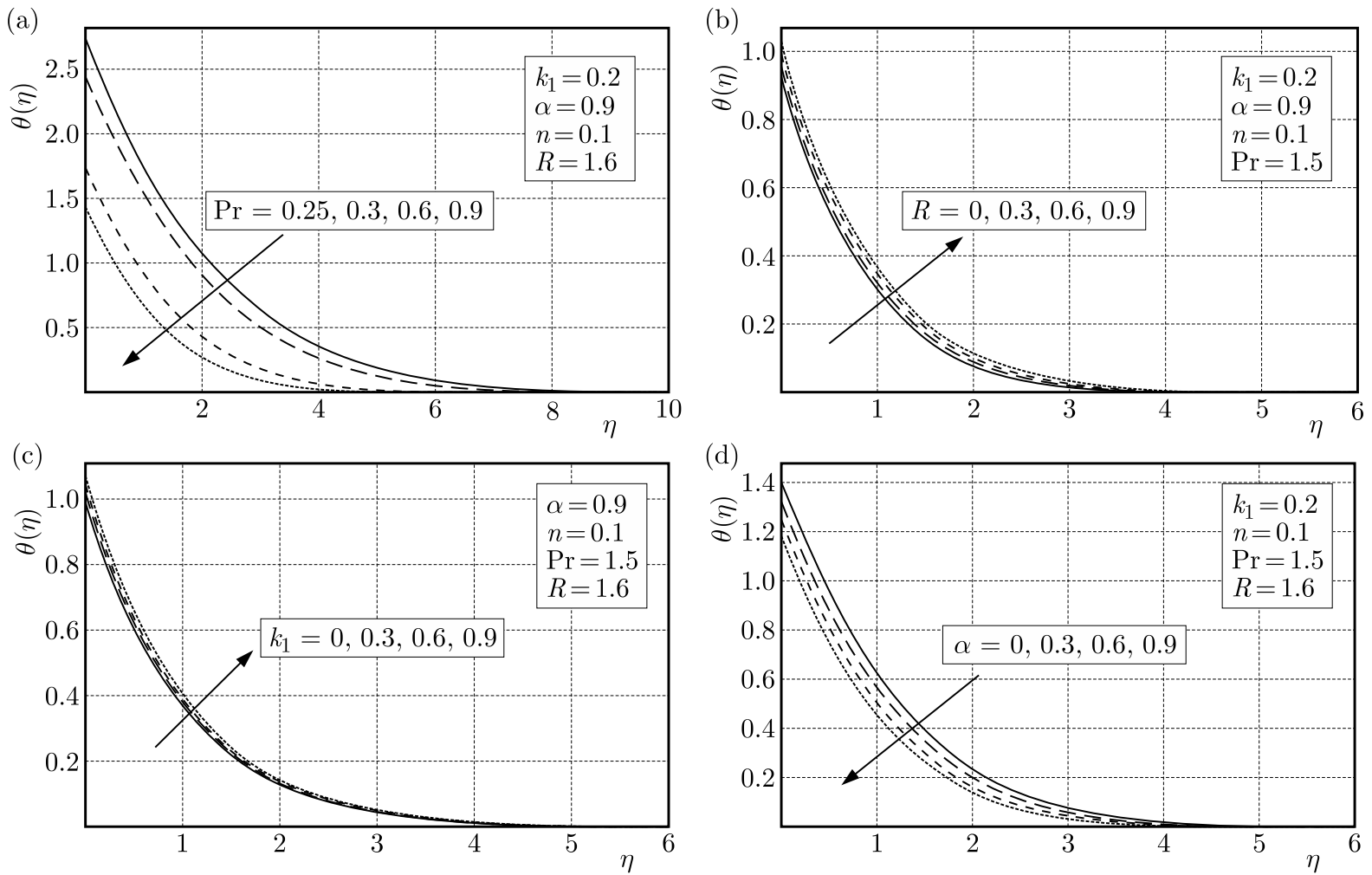

Fig. 3. Impact of (a) $\operatorname{Pr}$, (b) $R$, (c) $k_{1}$, (d) $\alpha$ 


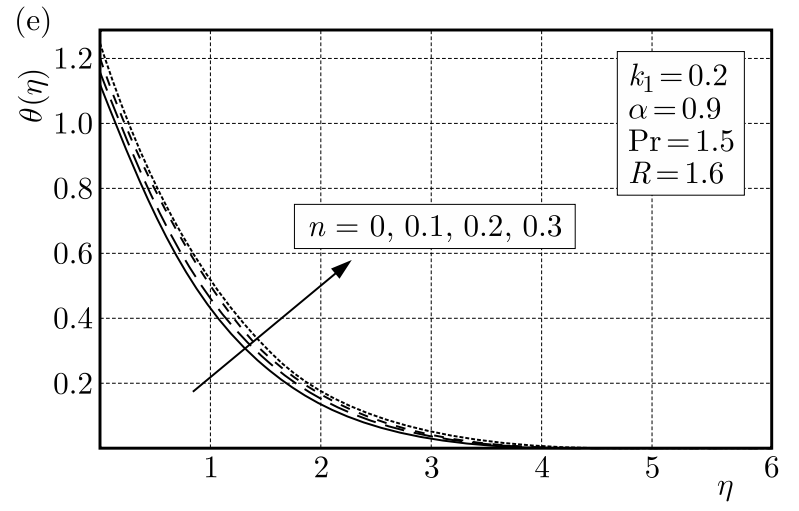

Fig. 4. Impact of $n$ on $\theta(\eta)$

Figure 3a shows the effect of $\operatorname{Pr}$ on the temperature profile. For increasing values of the Prandtl number, the temperature decreases. Higher values of Pr correspond to low thermal diffusivity, and the fluid temperature decreases. Figure $3 \mathrm{~b}$ depicts the behavior of fluid temperature for the radiation parameter $R$. This figure shows that the temperature profile enhances when radiation effects strengthen. An increase in the radiation parameter corresponds to a decrease in the mean absorption coefficient. Hence the rate of radiative heat transfer to the fluid increases. Figure 3c describes the behavior of temperature for viscoelastic parameter. Fluid temperature enhances for increasing $k_{1}$. Figure $3 \mathrm{~d}$ presents the effect of stretching ratio rates $\alpha$ on the temperature profile. The temperature profile shows decreasing behavior for increasing values of $\alpha$. The velocity increases when the ratio of stretching rates enhances. There is less resistance for fluid particles motion and, consequently, the temperature reduces. Figure 4 shows the effect of increasing values of $n$ on fluid temperature. The temperature profile and $n$ have a direct relation with each other.

\subsection{Nusselt number}

In this Section, we show the effects of different physical parameters on the Nusselt number. Figures 5a-5d depict the influence of the viscoelastic parameter $k_{1}$, Falkner-Skan power law index $n$, radiation parameter $R$ and Prandtl number Pr. These figures show that by increasing the viscoelastic and power law index parameters, the rate of heat transfer increases whereas the Nusselt number shows decreasing behavior for increasing values of the radiation parameter and the Prandtl number.

\section{Conclusions}

The Falkner-Skan wedge flow of Walter-B fluid is studied in presence of thermal radiation and prescribed surface heat flux. Key points of the presented analysis are as follows:

- Fluid velocity is a decreasing function of the viscoelastic parameter and increasing function of the ratio of stretching rates.

- The Prandtl number and radiation parameter have opposite impact on the temperature profile.

- For increasing values of the viscoelastic parameter, the temperature enhances.

- The Nusselt number has opposite impact on the power law index and the Prandtl number. 
(a)

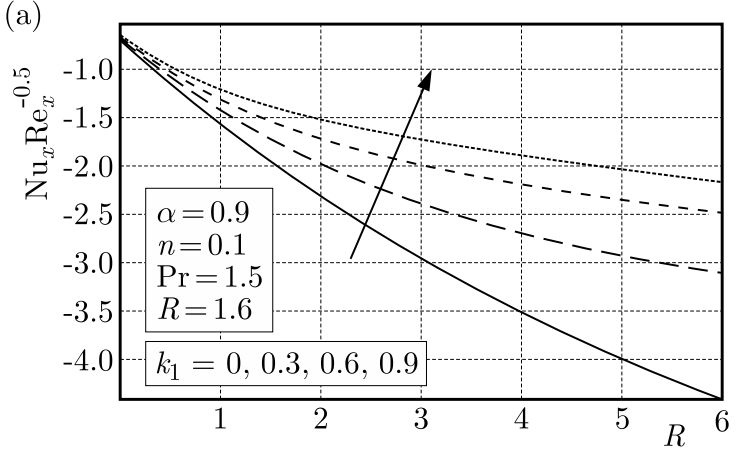

(c)

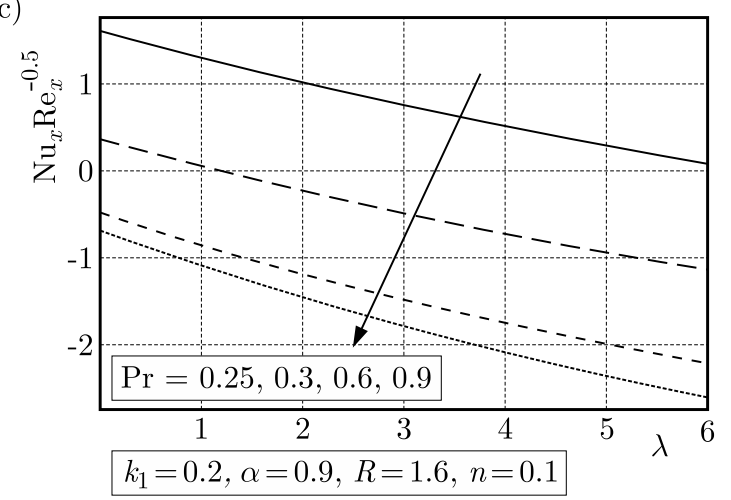

(b)

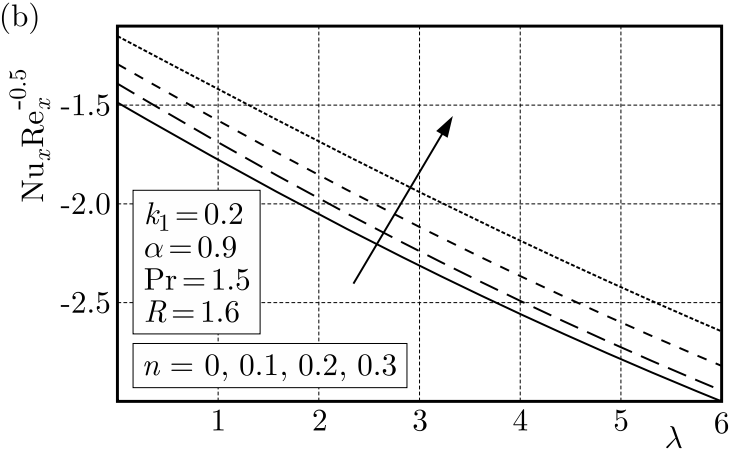

(d)

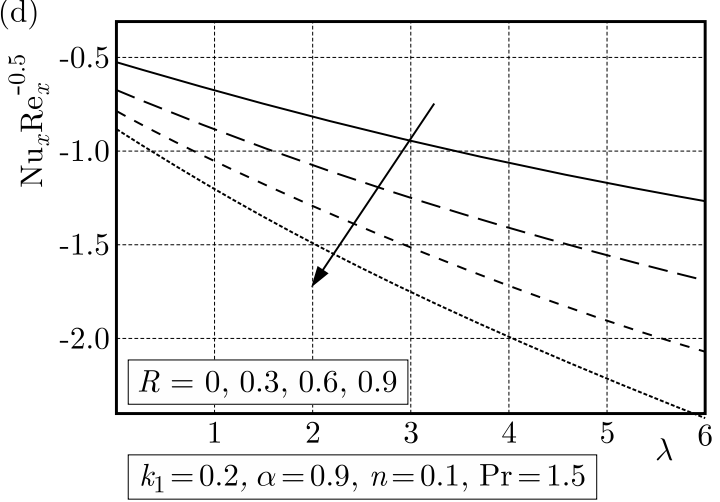

Fig. 5. Impact of (a) $k_{1}$, (b) $n$, (c) $R$ and (d) $\operatorname{Pr}$ on $\mathrm{Nu}_{x} \operatorname{Re}_{x}^{-0.5}$ (see Eq. (2.13))

\section{References}

1. Abbasbandy S., Hayat T.,Alsaedi A., Rashidi M. M., 2014a, Numerical and analytical solutions for Falkner-Skan flow of MHD Oldroyd-B fluid, International Journal of Numerical Methods of Heat and Fluid Flow, 24, 390-401

2. Abbasbandy S., Naz R., Hayat T., Alsaedi A., 2014b, Numerical and analytical solutions for Falkner-Skan flow of MHD Maxwell fluid, Applied Mathematics and Computation, 242, 569-575

3. Bhattacharyya K., 2013, MHD stagnation point flow of Casson fluid and heat transfer over a stretching sheet with thermal radiation, Journal of Thermodynamics, 2013, 169674

4. Bhattacharyya K., Mukhopadhyay S., Layek G.C., Pop I., 2012, Effects of thermal radiation on micropolar fluid flow and heat transfer over a porous shrinking sheet, International Journal of Heat and Mass Transfer, 55, 2945-2952

5. Chang T.B., Mehmood A., Beg O.A., Narahari M., Islam M.N., Ameen F., 2011, Numerical study of transient free convective mass transfer in a Walters-B viscoelastic flow with wall suction, Communications in Nonlinear Science and Numerical Simulation, 16, 216-225

6. FALKNER V.M., SkAN S.W., 1931, Some approximate solutions of the boundary-layer equations, Philosophical Magazine, 12, 865-896

7. Fang T., Yao S., Zhang J., Zhong Y., TaO H., 2012, Momentum and heat transfer of the Falkner-Skan flow with algebraic decay, Communications in Nonlinear Science and Numerical Simulation, 17, 2476-2488

8. Faroog U., Hayat T., Alsaedi A., Liao S. J., 2014, Heat and mass transfer of two-layer flows of third-grade nano-fluids in a vertical channel, Applied Mathematics and Computation, 242, $528-540$

9. Hakeem A.K.A., Ganesh N.V., Ganga B., 2014, Effect of heat radiation in a Walter's liquid B fluid over a stretching sheet with non-uniform heat source/sink and elastic deformation, Journal of King Saud University - Engineering Sciences, 26, 168-175 
10. Hayat T., Asad S., Mustafa M., Alsulami H.H., 2014a, Heat transfer analysis in the flow of Walters' B fluid with a convective boundary condition. Chinese Physics B, 23, 084701

11. Hayat T., Awais M., Asghar S., 2013a, Radiative effects in a three-dimensional flow of MHD Eyring-Powell fluid, Journal of Egyptian Mathematical Society, 21, 379-384

12. Hayat T., Farooq M., Iqbal Z., Alsaedi A., 2012, Mixed convection Falkner-Skan flow of a Maxwell fluid, Journal of Heat Transfer, 134, 114504

13. Hayat T., Imtiaz M., Alsaedi A., 2015a, MHD flow of nanofluid with homogeneousheterogeneous reactions and velocity slip, Thermal Sciences, DOI: 10.2298/TSCI140922067H

14. Hayat T., Imtiaz M., Alsaedi A., 2015b, Partial slip effects in flow over nonlinear stretching surface, Applied Mathematical Mechanics, 36, 1513-1526

15. Hayat T., Imtiaz M., Alsaedi A., 2016, Unsteady flow of nanofluid with double stratification and magnetohydrodynamics, International Journal of Heat and Mass Transfer, 92, 100-109

16. Hayat T., Naz R., Asghar S., Alsaedi A., 2014b, Soret-Dufour effects on MHD rotating flow of a viscoelastic fluid, International Journal of Numerical Methods of Heat and Fluid Flow, 24, 498-520

17. Hayat T., Shafiq A., Mustafa M., Alsaedi A., 2015c, Boundary-layer flow of Walters' B fluid with Newtonian heating, Zeitschrift für Naturforschung A, 70, 5, 333-341

18. Hayat T., Shahzad S. A., Asghar S., 2013b, MHD flow of thixotropic fluid with variable thermal conductivity and thermal radiation, Walailak Journal of Science and Technology, 10, 2942

19. Hayat T., Waqas M., Shehzad S. A., Alsaedi A., 2013c, Mixed convection radiative flow of Maxwell fluid near stagnation point with convective condition, Journal of Mechanics, 29, 403-409

20. Hendi F. A., Hussain M., 2012, Analytic solution for MHD Falkner-Skan flow over a porous surface, Journal of Applied Mathematics, 2012, 123185

21. Khan W.A., Pop I., 2013, Boundary layer flow past a wedge moving in a nano-fluid, Mathematical Problems in Engineering, 2013, 637285

22. Lin Y., Zheng L., Cheng G., 2015, Unsteady flow and heat transfer of pseudoplasticnanoliquid in a finite thin film on a stretching surface with variable thermal conductivity and viscous dissipation, Powder Technology, 274, 324-332

23. Madani S.A., Akbar R., Khoeilar R., 2012, On the study of viscoelastic Walter's B fluid in boundary layer flows, Mathematical Problems in Engineering, 2012, 861508

24. Nadeem S., Mehmood R., Motsa S.S., 2015, Numerical investigation on MHD oblique flow of Walter's B type nanofluid over a convective surface, International Journal of Thermal Sciences, 92, 162-172

25. Nandepanavar M.M., Abel M.S., Tawade J.V., 2010, Heat transfer in a Walter's liquid B fluid over an impermeable stretching sheet with non-uniform heat source/sink and elastic deformation, Communications in Nonlinear Science and Numerical Simulation, 15, 1791-1802

26. PAL D., 2013, Hall current and MHD effects on heat transfer over an unsteady stretching permeable surface with thermal radiation, Computer Mathematical with Applications, 66, 1161-1180

27. Ramesh K., Devakar M., 2015, Effect of heat transfer on the peristaltic flow of Walter-B fluid in a vertical channel with external magnetic field, Journal of Aerospace Engineering, 10, 04015050

28. Ramzan M., Faroog M., Alhothuali M.S., Malaikah H.M., Cui W., Hayat T., 2015, Three dimensional flow of an Oldroyd-B fluid with Newtonian heating, International Journal of Numerical Methods of Heat and Fluid Flow, 25, 1, 68-85

29. Rashidi M.M., Ali M., Freidoonimehr N., Rostami B., Hossain M.A., 2014, Mixed convective heat transfer for MHD viscoelastic fluid flow over a porous wedge with thermal radiation, Advance Mechanical Engineering, 6, 735939 
30. Shahzad S.A., Qasim M., Hayat T., Sajid M., Obaidat S., 2013, Boundary layer flow of Maxwell fluid with power law heat flux and heat source, International Journal of Numerical Methods of Heat and Fluid Flow, 23, 1225-1241

31. Sheikholeslami M., Ganji D.D., Javed M.Y., Ellahi R., 2015, Effect of thermal radiation on MHD nanofluid flow and heat transfer by means of two phase model, Journal of Magnetic Magnetism Material, 374, 36-43

32. Su X., Zheng L., 2011, Approximate solutions to MHD Falkner-Skan flow over permeable wall, Appllied Mathematical Mechanics, 32, 401-408

33. Sui J., Zheng L., Zhang X., Chen G., 2015, Mixed convection heat transfer in power law fluids over a moving conveyor along an inclined plate, International Journal of Heat and Mass Transfer, 85, 1023-1033

34. TAlla H., 2013, Numerical study of flow of Walter's liquid B over an exponentially stretching sheet, International Journal of Scientific and Research Publications, 3, 2250-3153

35. Yacob N.A., Ishak A., Nazar R., Pop I., 2011, Falkner-Skan problem for a static and moving wedge with prescribed surface heat flux in nano-fluid, International Communications in Heat and Mass Transfer, 38, 149-153

Manuscript received March 30, 2016; accepted for print May 10, 2016 